Please quote as: Rietsche, R. \& Söllner, M. (2017): The obvious and hidden values of IT-based peer assessment in management education. In: Academy of Management Annual Meeting (AOM). Atlanta, Georgia, USA. 
The obvious and hidden values of IT-based peer assessment in management education

\section{Authors}

Roman Rietsche ${ }^{1}$, Matthias Söllner ${ }^{1,2}$

${ }^{1}$ University of St. Gallen

Institute of Information Management

E-Mail: \{roman.rietsche, matthias.soellner\}@unisg.ch

${ }^{2}$ University of Kassel

Information Systems, Research Center for IS Design (ITeG)

E-Mail: soellner@uni-kassel.de 


\title{
The obvious and hidden values of IT-based peer assessment in management education
}

\begin{abstract}
Feedback is one of the most influential factor when it comes to learning success of students. Especially large-scale classes at universities often lack feedback caused by the scarcity of resources. Even though a lack of feedback is problematic across all domains, it is especially crucial when aiming to educate future managers, who are supposed to lead team members by providing effective feedback. In this study, we use problem-based-learning and a constructivist learning environment using IT-based peer assessment (ITPA) in a large-scale class to support future managers with feedback during the teaching-learning process. By implementing ITPA, we ensure on the one hand that our students regularly receive feedback, and one the other hand, we additionally support our students in training their ability to provide constructive feedback. While the results of our research show that the conspicuous value of ITPA is the increase of the students learning success and the hidden value is that at the same time the students train their ability to provide feedback. Specifically, our results show that participating in ITPA could lead to an increase in knowledge on the content of the class of about $28 \%$ on average. Furthermore, the students' ability to provide feedback - measured twice, self-reported and as quality of the feedback received - increased significantly during the semester.
\end{abstract}

Keywords: IT-based peer assessment, feedback, large-scale class, technology-mediated learning, management education 